American J. of Engineering and Applied Sciences 2 (4): 676-682, 2009

ISSN 1941-7020

(C) 2009 Science Publications

\title{
A New Engineering Method for Fuzzy Reliability Analysis of Surge Control in Centrifugal Compressor
}

\author{
Ahmed Hafaifa, Ferhat Laaouad and Moulod Guemana \\ Department of Industrial Process Automation, \\ Faculty of Hydrocarbons, University of Boumerdes, Algeria
}

\begin{abstract}
Problem statement: The surge phenomenon in the centrifugal compressor, the nonlinearities and uncertainties of the compression system make it impossible to use a conventional controller over a wide range of operation. Approach: A new dual fuzzy controller for nonlinear model of compression system was proposed in this study. This fuzzy controller was designed that consisted of active surge control and phase control without any explicit system models, but driven in human thinking mechanism. Results: Simulation example of compression system was given to demonstrate the validity of proposed control scheme. It was shown that fuzzy controller can be simplified and good tracking control performance can be achieved by choosing appropriate fuzzy roles. But, the dual fuzzy controller can successfully intervene in control surge of compression system. Conclusion: This new fuzzy control methodology suggested in this study reproduced well main characteristics of turbo compressor dynamic model developed by Moore and Gretzer and give place to a more precise and easy to handle representation. It is about an inaccuracies reproducing with a certain degree of satisfaction of real process without being as much complex.
\end{abstract}

Key words: Centrifugal compressor, anti surge control, fuzzy controller, safety systems

\section{INTRODUCTION}

Today the compression systems are subjected to highly hostile working conditions. The manufacturer is greatly interested with any improvement in performance, life and weight reduction without loss of reliability. Therefore, it is worthwhile to carefully estimate the reliability of rotating systems in order to improve the control system or eventually modify the design. Reliability analyses of the control structure require some information on the model of the compression system. We know it is difficult to obtain the mathematical model for a complicated mechanical structure. The turbo compressor is considered as a complex system where many modeling and controlling efforts have been made.

In the regard to the complexity and the strong non linearity of the turbo compressor dynamics and the attempt to find a simple model structure which can capture in some appropriate sense the key of the dynamical properties of the physical plant, we propose to study the application possibilities of the recent control approaches and evaluate their contribution in the practical and theoretical fields consequently. Facing to the studied industrial process complexity, we choose to make recourse to fuzzy logic for analysis and treatment of its control problem owing to the fact that these technique constitute the only framework in which the types of imperfect knowledge can jointly be treated (uncertainties, inaccuracies,...) offering suitable tools to characterize them. In the particular case of the turbo compressor, these imperfections are interpreted by modeling errors, the neglected dynamics and the parametric variations.

In 1965, Zadeh ${ }^{[5]}$ introduced fuzzy sets theory. Subsequently, the theory and the mathematics of fuzzy sets were fleshed-out and applied in many research fields ${ }^{[6]}$. In 1982, Takagi and Sugeno ${ }^{[10]}$ introduced a fuzzy rule-based model that can approximate a large number of nonlinear systems and the applications of fuzzy controller appear to have roots in many engineering fields ${ }^{[12]}$. The fuzzy logic intervenes efficiently in the surge control of the compression system. The fuzzy logic controller suggested in this study reproduced well the main characteristics of the turbo compressor dynamic model developed by Gretzer and Moore and give place to a more precise and easy to handle representation. It is about a inaccuracies reproducing with a certain degree of satisfaction of the real process without being as much complex ${ }^{[11]}$.

Corresponding Author: Ahmed Hafaifa, Department of Industrial Process Automation, Faculty of Hydrocarbons, University of Boumerdes, Algeria Tel: +213662140514 Fax: +21327873527 
Anti-surge control: The transfer of gas along a pipeline is a common process in the oil, chemical and petro-chemical industries. For cost-effectiveness, gas is usually transported at high pressure via a compressor before entering the pipeline. The compressor efficiency is maximized when the flow rate through it is kept low and the pressure high, with the minimum possible flow rate being restricted by the risk of compressor entering surge condition.

The surge phenomena is an unstable and undesirable operating condition of the compressor, occurring when the flow through it is reduced to the point where the compressor discharge pressure is less than the line pressure. This causes a momentary flow reversal, reducing line pressure and causing erratic flow output. With the reduced line pressure, flow through the compressor is re-established, causing line pressure to increase and the cycle to begin again. If the factors leading to the surge condition are not correctly and quickly rectified, the output will continue to oscillate resulting in damage to the compressor. The Anti-surge control offers:

- Protection against compressor damage such as bent shafts, cracked or ruptured castings, damaged impeller and bearings

- Reduction in compressor downtime and productivity costs

- Savings on maintenance costs

Although all anti-surge control techniques are based on a similar concept-to maintain a minimal flow at extreme conditions-fuzzy controller achieves this with a robust and efficient control module offering configuration and operator interface flexibility

If and when required by the process, the control module takes into account the following:

- Location of the flow measurement

- Type of compressor (axial, reciprocating or centrifugal)

- Type of operating speed (constant or variable)

- Value of discharge and suction pressures

- Value of inlet temperature

- Value of the compression ratio

- Composition of the transported gas (density, specific heat, molecular weight)

- Characteristics of all valves used in the control process

The compression system: Over fifteen years ago, Moore and Greitzer developed a phenomenological model for rotating stall and surge ${ }^{[6]}$. This pioneering work modeled the compression system with just three components. The first component is the inlet duct that allows infinitesimally small disturbances at the duct entrance to grow until they reach an appreciable magnitude at the compressor face. The second component is the compressor itself, modeled as an actuator disk, which raises the pressure ratio by doing work on the fluid. The third component is the plenum chamber (or diffuser) downstream, which acts as a large reservoir and responds to fluctuations in mass flow with fluctuations in pressure behind the actuator disk. In this study, we are considering a compression system consisting of a centrifugal compressor, close coupled valve, compressor duct, plenum volume and a throttle. The throttle can be regarded as a simplified model of a turbine $^{[1,13]}$. The system is showed in Fig. 1.

The model to be used for controller design is in the form:

$$
\left\{\begin{array}{l}
\dot{\mathrm{P}}_{\mathrm{p}}=\frac{\mathrm{kP}_{01}}{\rho_{01} \mathrm{~V}_{\mathrm{p}}}\left(\mathrm{m}-\mathrm{k}_{\mathrm{t}} \sqrt{\mathrm{P}_{\mathrm{p}}-\mathrm{P}_{01}}\right) \\
\dot{\mathrm{m}}=\frac{\mathrm{A}_{1}}{\mathrm{~L}_{\mathrm{c}}}\left[\mathrm{P}_{01}\left(1+\eta_{\mathrm{i}}(\mathrm{m}, \mathrm{N}) \frac{\Delta \mathrm{h}_{\text {ideal }}}{\mathrm{C}_{\mathrm{p}} \mathrm{T}_{01}}\right)^{\frac{4(\mathrm{k}-1)}{\mathrm{k}}}-\mathrm{P}_{\mathrm{p}}\right] \\
\dot{\mathrm{N}}=\frac{1}{2 \mathrm{~J} \pi}\left(\frac{\eta_{\mathrm{t}} \mathrm{m}_{\text {tur }} \mathrm{C}_{\mathrm{p}, \mathrm{t}} \Delta \mathrm{T}_{\text {tur }}}{2 \pi \mathrm{N}}-2 \mathrm{r}_{22} \sigma \pi \mathrm{N}|\mathrm{m}|\right)
\end{array}\right.
$$

Where:

$\mathrm{m}=$ The compressor mass flow

$\mathrm{p}_{\mathrm{p}}=$ The pressure downstream of the compressor

$\mathrm{a}_{01}=$ The inlet stagnation sonic velocity

$\mathrm{L}_{\mathrm{c}}=$ The length of compressor and duct

$\mathrm{A}_{1}=$ The area of the impeller eye (used as reference area)

$\mathrm{N}=$ The spool moment of inertia. The two first Equations of (1) are equivalent to the model of ${ }^{[4]}$.

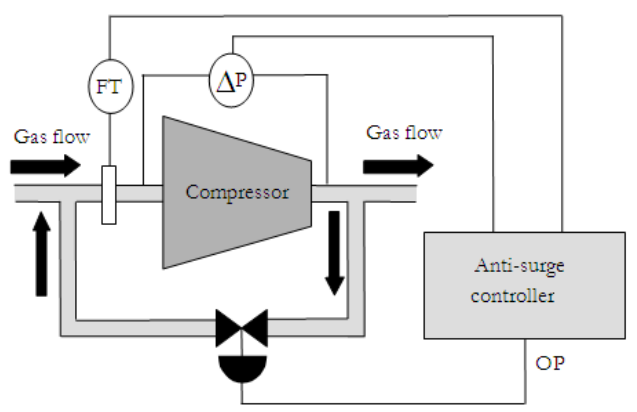

Fig. 1: The compression system 


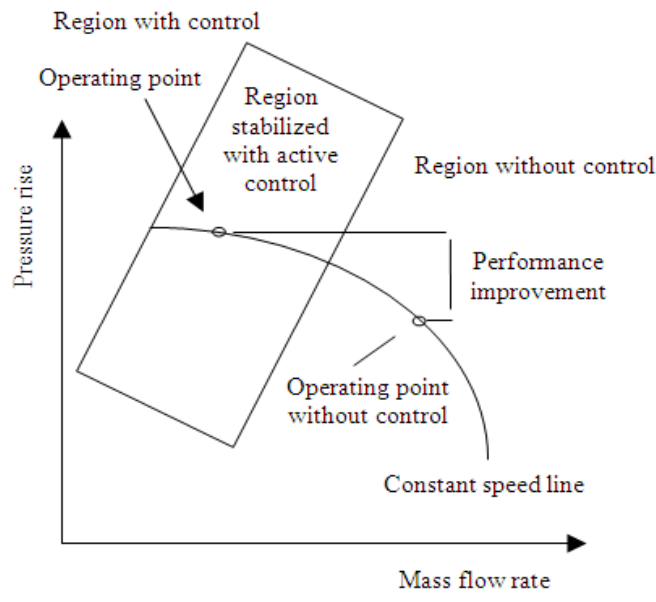

Fig. 2: Compressor performance characteristic map

The Moore-Greitzer model gives rise to three ordinary differential equations, the first for the nondimensional total-to-static pressure rise $\Delta \mathrm{p}$ across the compression system, the second for the amplitude of mass flow rate fluctuations $\mathrm{m}$ and the third for the nondimensional, spool moment of inertia. In the above equations, $\sigma$ and $\beta$ are constants that are characteristics of the system. The quantity $\phi_{\mathrm{T}}$ determines how much mass will be removed in a user-controlled fashion through a bleed valve. It may be written as ${ }^{[3]}$ :

$$
\phi_{\mathrm{T}}=\gamma \sqrt{\Delta \mathrm{P}}
$$

The functional form between $\phi$ and $\psi$ is simply the performance map and is often approximated by ${ }^{[9]}$ :

$$
\psi=\mathrm{a}+\mathrm{b} \phi+\mathrm{c} \phi^{2}+\mathrm{d} \phi^{3}
$$

where, a, b, c and d are constants which must be determined by a curve fit of the experimental data. The most important approximations underlying the MooreGreitzer model are that (i) it is valid under small perturbations $m$ and (ii) the time scale of the dynamics governing $\mathrm{m}$ is much faster than the time scale of the dynamics governing $\phi$.

The present study has analytically integrated the right hand side of Eq. 1. This integration does not require a priori assumptions about the analytical form of the performance map ${ }^{[7,8]}$.

Note that the Moore-Greitzer model does not attempt to explain what physical mechanism triggers these instabilities. Rather, it attempts to determine the favorable conditions under which the disturbances will grow and what can be done to suppress the instabilities. Its simplicity, mathematical elegance and generality have led to wide acceptance and use of this model by researchers in industry, government and academia. It is also used in surge control research with the belief that rotating stall is a precursor to surge and with the expectation that elimination of rotating stall will also eliminate the development of surge.

The instabilities within compression systems can be studied using energy considerations. As shown by Gysling and Greitzer ${ }^{[14]}$ the rate of energy input by the compressor to the fluid (over and above the steady state input) may be written as:

$\delta \mathrm{E}=\int_{\text {Annulus }} \delta(\Delta \mathrm{P}) \delta \phi \mathrm{dA}$

If this integral is positive, energy is added to the fluid by the compressor and the disturbances will grow in amplitude. In the performance map shown in Fig. 2, the slope of the curve is negative to the right side of the peak. In this region, a small increase in mass flow rate $\delta \phi$ will decrease pressure, so that $\delta(\Delta p)$ is negative.

Many studies and texts on anti-surge control maintain that the onset of surge can occur in as little as $50 \mathrm{~ms}^{[2,16]}$. They then conclude that this and the requirement for very "tight" tuning, implies that a digital anti-surge controller must have an extremely fast repeat time. Compressor users, however, point out that the blow off or recycle valve driven by the controller is unlikely to open in less than $2 \mathrm{sec}$. The application of fuzzy logic to the anti-surge control module has been proven to successfully meet the above criteria with repeat times of 75-100 ms.

The application module processes the pressure difference to calculate the correct flow set point. Two different sets of time constants are used in the fuzzy controller. For a positive deviation, where the flow rate is too high, the output signal to the flow control element produces a gradual correction to allow time for the control system to react, thus avoiding over-correction and risk of surge. If there is a negative deviation from the set point, a different set of time constants is used to open the by-pass valve quickly. This allows more gas to re-circulate, increasing the flow rapidly enough to avoid surge conditions.

In order to avoid turbulence, the application module also monitors the rate of change of the flow through the compressor. If the flow rate begins to change rapidly, the output to the flow control element by-passes the control loop and opens the valve fully. This allows the maximum amount of gas to re-circulate and increase the flow through the compressor. In general, the control system should be tuned so that the valve is shut most of the time, since re-circulating the gas consumer's energy. 
The different types of surge can be defined by the work region of the centrifugal compressor. As it can be shown in Fig. 3a-d the subjective feeling of surge as a result of a mass flow coefficient and pressure coefficient, is defined in a deep surge or as a Mild surge. In this way, the uncertainty present in every compression systems can be implicitly represented ${ }^{[15]}$.

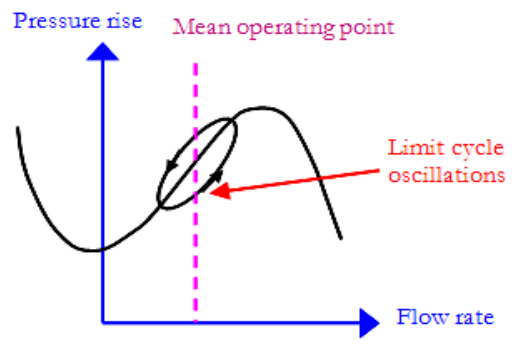

Fig. 3a: Mild surge

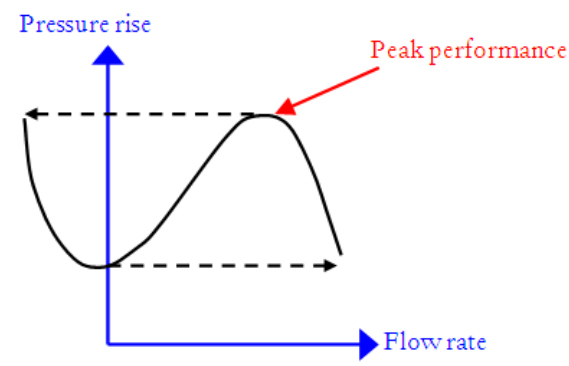

Fig. 3b: Deep surge

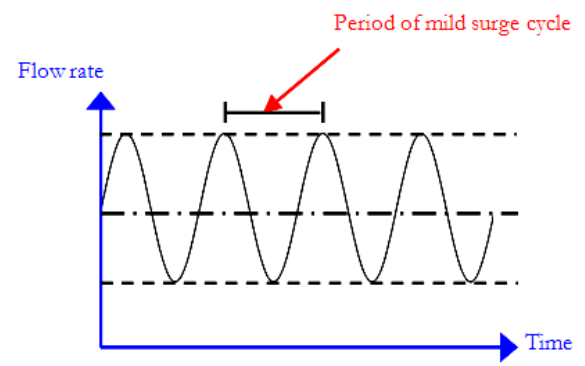

Fig. 3c: Mild surge of the flow rate

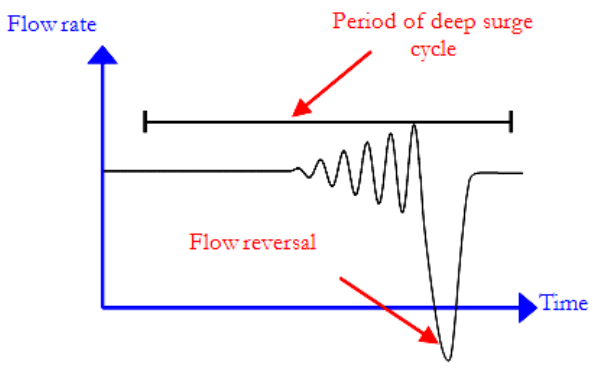

Fig. 3d: Deep surge of the flow rate

\section{MATERIALS AND METHODS}

Experimental data: This study presents results from previous tests and background studies; a review of published materials and recent laboratory tests funded by the Department of Automatic Control, of the University of Boumerdes, Algeria, in collaboration with the station of the gas compression of SONATRACH Algeria. Previous tests and the most recently available measured surge internal flow data is parameterized to help identify factors that affect the indications that a compressor is approaching surge.

Fuzzy control of compression system: The fuzzy logic control is a rule-based system that receives information fed back from the plant's operating, in this case the normalized fluctuations of $\Phi$ (the masse flow coefficient) and $\Psi$ (the pressure coefficient). These crisp values are fuzzified and processed using the fuzzy knowledge base. The fuzzy output is defuzzified in throttle and CCV gains in order to control the plants operating conditions. A fuzzy system involves identifying fuzzy inputs and outputs, creating fuzzy membership functions for each, constructing a rule base and then deciding what action will be carried out (Fig. 4).

The response of the system is used to model the control system. Increasing either the throttle gain $\gamma_{\mathrm{r}}$ or CCV gain $\gamma_{v}$ will stabilize the system with a penalty of pressure lost across the plenum. The fluctuations of the mass flow coefficient $\Delta \Phi$ and pressure coefficient $\Delta \Psi$ are normalized before being sent to the fuzzy model as the crisp input by the following:

$$
\begin{aligned}
& \Delta \Psi_{\mathrm{i}}=\frac{\left|\Psi_{\mathrm{i}}-\Psi_{\mathrm{i}+\Delta t}\right|}{\operatorname{Max}\left(\Psi_{\mathrm{i}}, \Psi_{\mathrm{i}+\Delta t}\right)} \\
& \Delta \Phi_{\mathrm{i}}=\frac{\left|\Phi_{\mathrm{i}}-\Phi_{\mathrm{i}+\Delta t}\right|}{\operatorname{Max}\left(\Phi_{\mathrm{i}}, \Phi_{\mathrm{i}+\Delta t}\right)}
\end{aligned}
$$

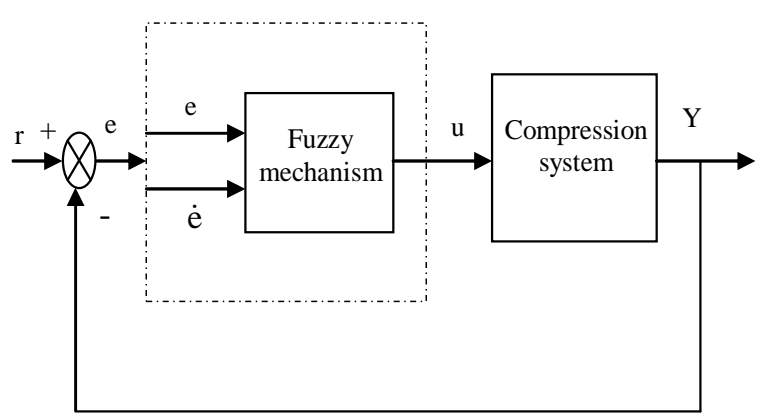

Fig. 4: Bloc diagram of the fuzzy control system. 
Samples of the coefficients are taken at regular time-step intervals:

$$
\Delta \mathrm{t}=\mathrm{kh}
$$

Where:

$\mathrm{k}$ is a constant

$\mathrm{h}$ is the Runge-Kutta time step size

The crisp output from the fuzzy model adjusts both control gains by the following:

$\gamma_{i+\Delta t}=\gamma_{i}+\gamma_{i} \Delta \gamma_{i}$

Triangular membership functions are defined for each classified category of input and output of the compression system. The base of each triangular membership function rests on the intervals of each category and the apex of the triangle is located above the midpoint of the interval.

\section{RESULTS}

The results of tows simulations are presented in this section. The first is the results of simulations of the compression system in low back pressure and the high back pressure nearing surge at $15 \mathrm{KRPM}$ of compressor speed and the simulations of the compression system without control of surge and the second simulation is the compression system with control of surge using fuzzy logic controller. The response of the compression system in low back pressure at 15 KRPM of compressor speed is shown in Fig. 5 and the response of the system without control of surge is shown in Fig. 6.
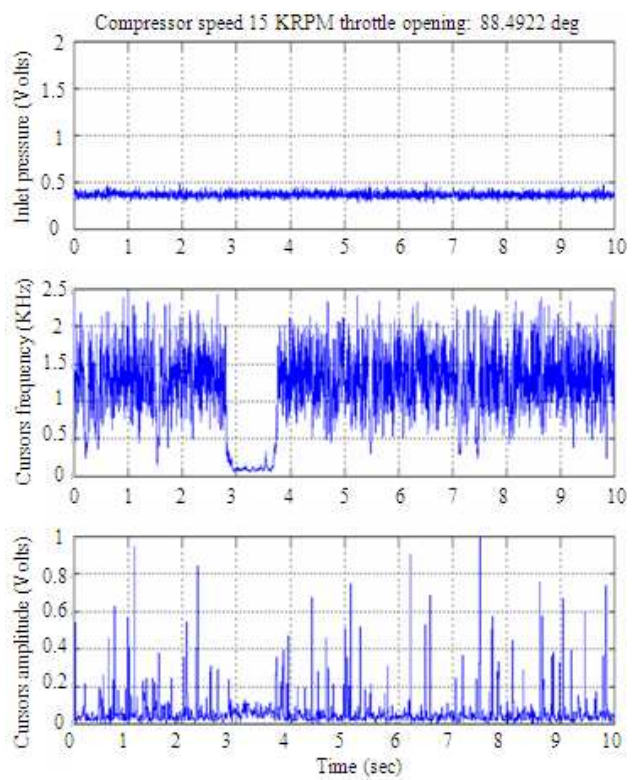

Fig. 5: Low back pressure, 15 KRPM
The response of the compression system in High back pressure nearing surge at $15 \mathrm{KRPM}$ of compressor speed is shown in Fig. 7and the response of the system without control of surge is shown in Fig. 8.

The response of the compression system with control of surge using fuzzy logic controller is shown in Fig. 9 and the response of the compression system with Control Using Fuel Valve Actuation is shown in Fig. 10 fuzzy logic controller of the compression system was constructed using data from the simulation model.
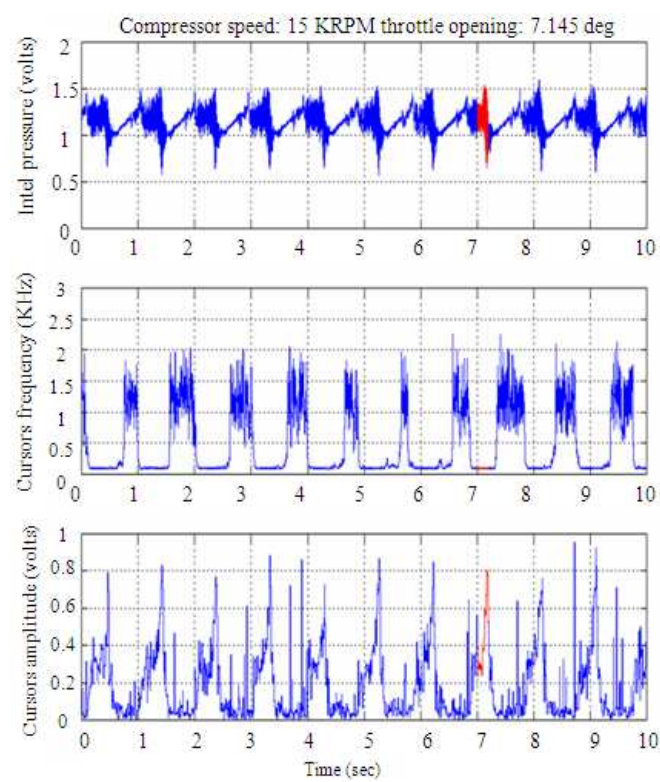

Fig. 6: Uncontrolled surge, 15 KRPM
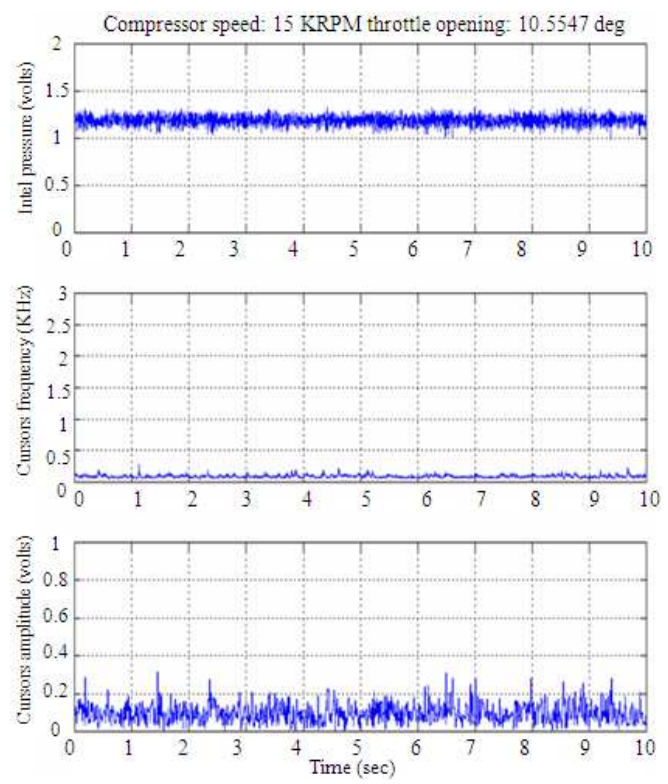

Fig. 7: High back pressure nearing surge, 15 KRPM 680 

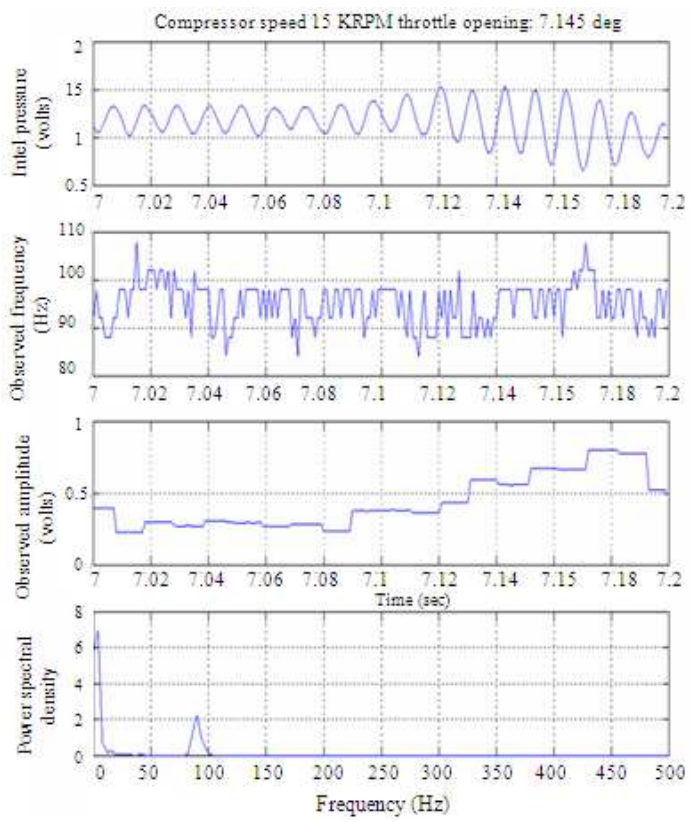

Fig. 8 Uncontrolled surge, 15 KRPM
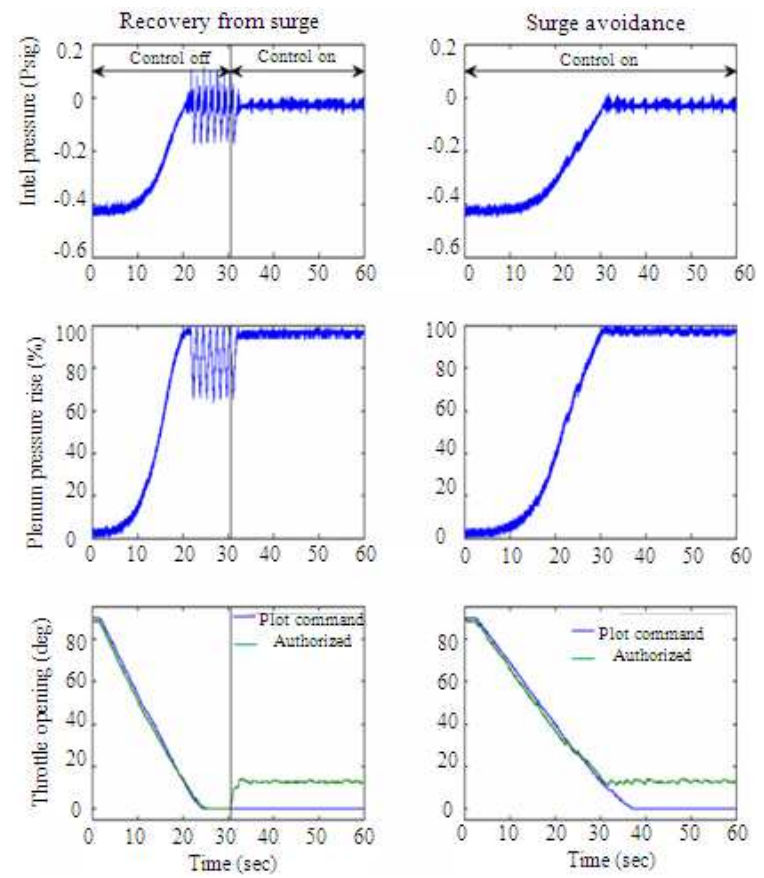

Fig. 9: The response of the compression system with control of surge using fuzzy logic control

Then through the optimization routine, the model predictive controller provides a control action to the system depending on the predictive horizon and the control weighting factor.
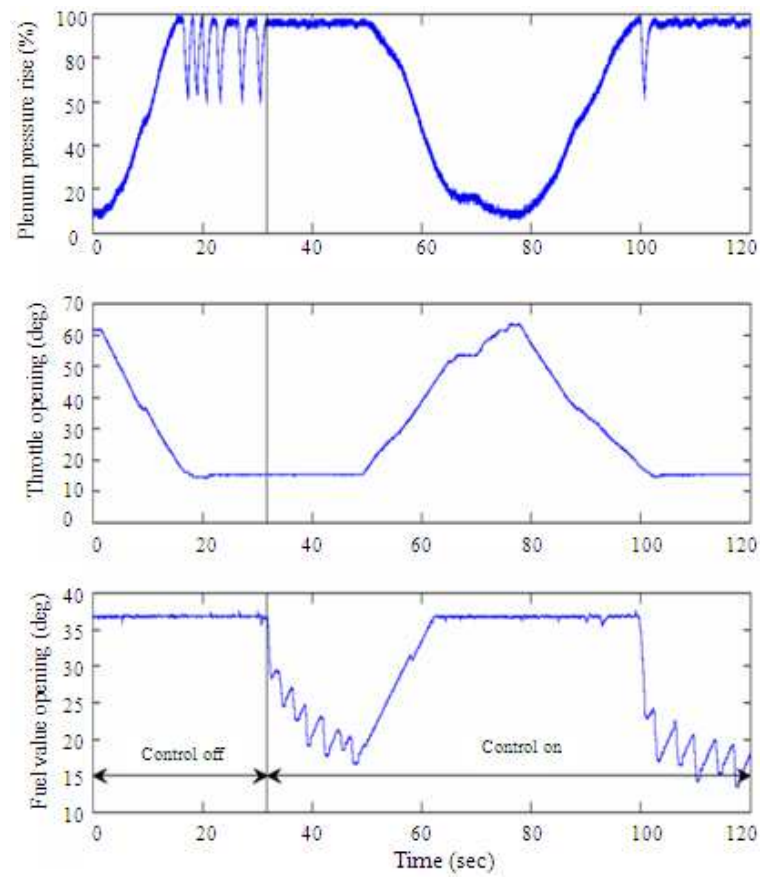

Fig. 10: The response of the compression system with using fuel valve actuation

\section{DISCUSSION}

Fuzzy logic of different complexities were studied, the larger the computational time but also the better the results. A model predictive controller with a longer prediction horizon and a small control weighting factor provides good performance in terms of anti surge control and reduced error. However, the observation on the variation of the controller output provided an interesting result. Implementing such a controller on a real-time system would probably be prohibitive due to the fact that there are limitations on the incremental variation of the compression system.

\section{CONCLUSION}

The study has shown, firstly how a fuzzy logic may be trained to approximate an existing nonlinear controller. The development of independent fuzzy logic control with model predictive controller presented a further step in the research. In theory, predictive control, discussed here only, would probably provide benefits for a compression system. Use of fuzzy logic controller, however, could provide a good compromise as the training of the fuzzy logic controller is done offline. Performances in terms of anti-surge control system, overshoot and time to reach the set point are very similar to those of the PID controller. 
The proposed dual fuzzy controller successfully performed the surge control as well as the active control of a centrifugal compressor. No derivation of mathematical models is needed and no system identification is required for the implementation of fuzzy controllers, in which the fuzzy control law is robust to the values of membership functions, disturbances and loads. Although the compression system inheres a narrow operating range with a bandwidth around $14 \mathrm{~Hz}$, the dual fuzzy controller can even drive the centrifugal compressor to oscillate up to $20 \mathrm{~Hz}$ with satisfactory tracking performance in the surge control. This research result may be applied to various compression systems.

\section{REFERENCES}

1. Burnham, K.J., A. Dunoyer and S. Marcroft, 1999. Bilinear controller with PID structure. Comput. Control Eng. J., 10: 63-69. http://ieeexplore.ieee.org/xpl/freeabs_all.jsp?tp=\&a rnumber $=768662 \&$ isnumber $=16635$

2. Draincov, D., H. Hellendoorn and M.R. Frank, 1996. An introduction to Fuzzy Control. SpringerVerlag, ISBN: 3540606912, pp: 316.

3. Dien Z., Min K.C., 1995. Fuzzy Logic and Its Applications to Engineering, Information Sciences and Intelligent Systems. 1st Edn., Kluwer Academic Publishers, ISBN: 10: 0792337557, pp: 496.

4. Gager, D. and J.T Gravdahl, 1995. Rottating stall and surge control. Proceedings of the 35th Conference on Decision and Control, Dec. 13-15, IEEE Xplore Press, New Orleans, pp: 1857-1862. DOI: 10.1109/CDC.1995.480612

5. Gysling, D.L. and E.M. Greitzer, 1995. Dynamic control of rotating stall in axial flow compressors using aeromechanical feedback. J. Turbomach., 117: 307-319.

http://cat.inist.fr/?aModele $=$ afficheN\&cpsidt $=3643234$

6. Laaouad, F., Hafaifa A. and Laroussi K., 2007. A nonlinear model for a turbo compressor using fuzzy logic approach Proceedings of the 6th WSEAS International Conference on Electronics, Hardware, Wireless and Optical Communications. Feb. 16-19, Corfu Island, Greece, pp: 79-84. http://www.wseas.us/e-

library/conferences/2007corfu/papers/541-396.pdf

7. Laaouad, F., H. Fekhar and A. Hafaifa, 2002. Adaptive control of centrifugal compressors. Proceedings of the 15th International Congress of Chemical and Process Engineering, Apr. 2007, Prague, Tchequie, pp: 168-171.
8. Laaouad, F., H. Fekhar and A. Hafaifa, 2003. Conception of new control systems using neural networks and fuzzy logic in chemical and petrochemical plants. Proceedings of the 7th International Conference on Chemistry and Its Role in Development, (ICCIRD'03), Egypt, pp: 233-241.

9. Lerat, A., J. Sidès and V. Daru, 1982. An implicit finite-volume method for solving the Euler equations. Lecture Note Phys., 170: 343-349. DOI: 10.1007/3-540-11948-5_41

10. Lee, R., 1990. Fuzzy logic in control system: Fuzzy logic controller. Part I. IEEE. Trans. Syst. Man Cybernet., 20: 419-353. DOI: $10.1109 / 21.52552$

11. Leonessa, A., V. Chellaboina and W.M. Haddad, 1997. Globally stabilizing controllers for multimode axial flow compressor models via equilibriadependent lyapunov functions. Proceedings IEEE Conference Controls Applied, Oct. 5-7, Albuquerque NM., USA., pp: 63-68. DOI: 10.1109/CCA.1997.627472

12. Leonessa, A., V. Chellaboina and W.M. Haddad, 1997. Robust stabilization of axial flow compressors with uncertain pressure-flow maps. IEEE Trans. Control Syst. Technol., 8: 466-473. http://direct.bl.uk/bld/PlaceOrder.do?UIN=079871321\& $\mathrm{ETOC}=\mathrm{RN} \&$ from $=$ searchengine

13. Liaw, D.C. and E.H. Abed, 1992. Stability analysis and control of rotating stall. IFAC Nonlinear Control System Design, Bordeaux, France, pp: 295-300. http://direct.bl.uk/bld/PlaceOrder.do?UIN=008924 030\&ETOC $=$ EN\&from $=$ searchengine

14. Moore, F.K. and E.M. Greitzer, 1986. A theory of post-stall transients in axial compressor systems. II: Application. J. Turbomach. Gas Turbines Power, 108: 231-239. http://cat.inist.fr/?aModele=afficheN\&cpsidt=7882599

15. Minihan, J.A., Burnham K.J., Dunoyer A. and Hibberd D.F. 1999. A bilinear controller with PID structure. Proceedings of the American Control Conference, San Diego, CA, USA pp: 4363-4367. DOI: 10.1109/ACC.1999.786392

16. Markopoulos, N., Y. Neumeier, J.V.R. Prasad and B.T. Zinn, 1998. Active control of compressor rotating stall using linear amplitude feedback. Proceedings of the 36th Aerospace Sciences Meeting and Exhibit, Jan. 12-15, Reno, NV., pp: 98-971.

http://www.aiaa.org/content.cfm?pageid=406\&gTa ble $=$ mtgpaper $\& \mathrm{gID}=17070$ 